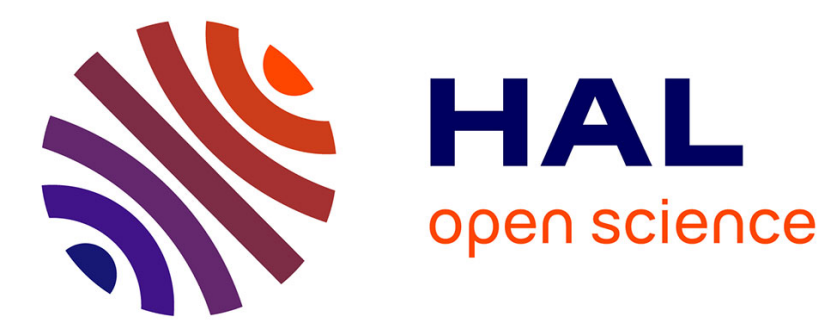

\title{
Stabilizing effect of cannibalism in a two stages population model
}

\author{
Jonathan Rault, Eric Benoît, Jean-Luc Gouzé
}

\section{To cite this version:}

Jonathan Rault, Eric Benoît, Jean-Luc Gouzé. Stabilizing effect of cannibalism in a two stages population model. Acta Biotheoretica, 2013, 61, pp.119-139. 10.1007/s10441-013-9172-x . hal-00848443

\section{HAL Id: hal-00848443 \\ https://hal.inria.fr/hal-00848443}

Submitted on 7 Aug 2013

HAL is a multi-disciplinary open access archive for the deposit and dissemination of scientific research documents, whether they are published or not. The documents may come from teaching and research institutions in France or abroad, or from public or private research centers.
L'archive ouverte pluridisciplinaire HAL, est destinée au dépôt et à la diffusion de documents scientifiques de niveau recherche, publiés ou non, émanant des établissements d'enseignement et de recherche français ou étrangers, des laboratoires publics ou privés. 


\title{
Stabilizing effect of cannibalism in a two stages population model
}

\author{
Jonathan Rault • Eric Benoît • Jean-Luc \\ Gouzé
}

Received: date / Accepted: date

\begin{abstract}
In this paper we build a prey-predator model with discrete weight structure for the predator. This model will conserve the number of individuals and the biomass and both growth and reproduction of the predator will depend on the food ingested. Moreover the model allows cannibalism which means that the predator can eat the prey but also other predators. We will focus on a simple version with two weight classes or stage (larvae and adults) and present some general mathematical results. In the last part, we will assume that the dynamics of the prey is fast compared to the predator's one to go further in the results and eventually conclude that under some conditions, cannibalism can stabilize the system: more precisely, an unstable equilibrium without cannibalism will become almost globally stable with some cannibalism. Some numerical simulations are done to illustrate this result.
\end{abstract}

Keywords Stage-structured model · prey-predator model · Cannibalism · Slow-fast systems

J. Rault

INRIA BIOCORE

2004 Route des Lucioles B.P. 93, 06902 Sophia Antipolis CEDEX, France

E-mail: jonathan.rault@inria.fr

E. Benoît

Laboratoire de Mathématiques, Université de La Rochelle

Avenue Michel Crépeau, 17042 La Rochelle, France

E-mail: ebenoit@univ-lr.fr

J.L. Gouzé

INRIA BIOCORE

2004 Route des Lucioles B.P. 93, 06902 Sophia Antipolis CEDEX, France

E-mail: jean-luc.gouze@inria.fr 


\section{Introduction}

Size-structured or weight-structured models are more and more used in biological modelling because they allow more realism in the described phenomena. In population dynamics, the structure can be age, size, weight or even any physiological parameter that plays an important role in the behaviour of individuals (Tuljapurkar and Caswell, 1997).

Such models with continuous size or weight structure are particularly used for fish and zooplankton dynamics (see e.g. (Benoît and Rochet, 2004; Maury et al, 2007)). The dynamics described in these models often are the dynamics of an aggregation of species and since in the model it is not possible to differentiate one specie from another, size-dependent predation can exactly be interpreted as cannibalism. Moreover, actual cannibalism is also present in some zooplankton species where larger individuals can eat the resource but also smaller individuals of the same species (Gallucci and Ölafsson, 2007). Cannibalism can also occur in some species of spiders and mantis where female eats male after mating to increase the eggs production (Birkhead et al, 1988; Elgar and Nash, 1988).

The aim of this article is to show that cannibalism can have a stabilizing effect in the population dynamics (see (Claessen et al, 2004) for a short review of cannibalism studies in mathematical models). The mathematical study of cannibalism in continuous weight structured models can be rather complex, and in most cases only a linearized study is possible ((Farkas and Hagen, 2007)). To bring to light the effect of cannibalism we will first build in section 2 a prey-predator model with discrete weight-structure for the predator: the system obtained is a system of ordinary differential equations, more amenable to global studies. This model itself is, to our knowledge, original, and can also be seen as a stage-structured model for which a weight is associated with each stage. It includes cannibalism and preserves the total biomass and number of individuals; the passage from a stage to the following one and the reproduction will be food ingestion dependent (see (de Roos et al, 2008) for related work). Stabilizing (or destabilizing) effect of cannibalism in discrete models has been studied in (Kaewmanee and Tang, 2003; Kohlmeier and Ebenhöh, 1995; Magnússon, 1999) and conclusions obtained can be opposite and actually seem to be strongly dependent on the model.

To study the model we built, we will focus on a simple version with two weight classes for the predator; cannibalism will occur only throughout the adult populations. Its level will be parametrized by a parameter $\kappa$ between 0 and 1 (one corresponds to no cannibalism). In section 4 we will suppose that there is two different time scales. The dynamics of the prey will be faster than the dynamics of the predator (this hypothesis is classical), and will allow us to simplify further the system. For some mathematical reference on singular perturbation and slow-fast system see (Benoît, 1983; O'Malley, 1991) and (Liu et al, 2003; Rinaldi and Muratori, 1992) for examples of application in biology context.

We show using this slow-fast system approach that under some hypotheses 
the equilibrium without cannibalism is unstable, and we prove the existence of periodic oscillations (limit cycle) for the reduced system. The equilibrium becomes stable and the oscillations disappear if cannibalism occurs, below some threshold. We are able to study the slow-fast system in a global way. We end by some simulations and a bifurcation diagram.

\section{Model description}

\subsection{Biological assumptions}

In this section we will build a general version of the model with $M$ weight classes for the predator. Each class has a number $N_{i}(t)$ of individuals each one of weight $m_{i}$. The total biomass of the class $i$ is then $m_{i} N_{i}(t)$. The biological hypotheses we make are the following:

(H1) Weight of individuals is an increasing function of class and so $m_{i}<m_{j}$ for $i<j$.

(H2) Each individuals of a given class can feed on resource and/or other predators of various classes (if they are cannibals). When they feed, only a fraction $k_{a}$ of the acquired biomass is assimilated and can be used, the other fraction is lost in the system.

(H3) Assimilated biomass of individuals that are not in the final class is used to grow and therefore permits the passage from a class to the following one.

(H4) Assimilated biomass of individuals that are in the final class is used to lay. A fraction $\epsilon$ of the laid eggs hatch and become individuals of the first class.

(H5) Each weight class has its own mortality rate, which is strictly positive and not depending on time. This mortality rate include natural mortality and external mortality due to super predators.

(H6) Biomass conservation and conservation of the number of individuals hold during all these processes and interactions between individuals.

Introduce the vector $N=\left(N_{1}, \ldots, N_{M}\right)$. The biomass $f_{i}$ acquired per unit of time by an individual of the weight class $i$ is composed of resource (function $f_{i, R}$ in $\left.(2.1)\right)$ and functions $f_{i, j}$ that come from cannibalism on the weight class $j$ and that can be equal to zero if we suppose no cannibalism on weight class $j$ by the individual of class $i$. These quantities can (a priori) depend on the current state of the system i.e. of $R$ and $N$. The assimilated biomass is then a fraction $\left.\left.k_{a} \in\right] 0,1\right]$ of the biomass that has been eaten. We obtain:

$$
k_{a} \cdot f_{i}(R, N)=k_{a} \cdot\left(f_{i, R}(R, N)+\sum_{j=1}^{M} f_{i, j}(R, N)\right)
$$


This predation of individuals of the class $i$ on the class $j$ leads to a (total) mortality rate in the class $j$ :

$$
\mu_{j, i}(R, N)=\frac{f_{i, j}(R, N)}{m_{j}} N_{i}
$$

The mortality rate of the class $j$ resulting from the whole cannibalism within predators is then:

$$
\mu_{j}(R, N)=\frac{1}{m_{j}} \sum_{i=1}^{M} f_{i, j}(R, N) N_{i}
$$

Remark 1 It can be biologically realistic to suppose that cannibalism can occur only on smaller weight classes. It would result that $f_{i, j}(R, N)=0$ for $j>i$.

Knowing the assimilated biomass per unit of time, the question we now have to answer concerns the flux of individuals going from class $i$ to class $i+1$ (when $i<M$ ). Leaving out all other mechanisms (external mortality, cannibalism mortality and individuals arriving in class $i$ from the class $i-1$ ), biomass conservation and number of individuals conservation give us:

$$
\begin{aligned}
& \dot{N}_{i}+\dot{N}_{i+1}=0 \\
& m_{i} \dot{N}_{i}+m_{i+1} \dot{N}_{i+1}=k_{a} f_{i}(R, N) N_{i}
\end{aligned}
$$

It results that:

$$
\dot{N}_{i+1}=-\dot{N}_{i}=\frac{k_{a}}{m_{i+1}-m_{i}} f_{i}(R, N) N_{i}
$$

which yields considering all fluxes that: for $i \in\{2, \ldots, M-1\}$,

$$
\begin{aligned}
\dot{N}_{i}= & \underbrace{\frac{k_{a}}{m_{i}-m_{i-1}} f_{i-1}(R, N) N_{i-1}}_{\text {arriving from class } i-1}-\underbrace{\frac{k_{a}}{m_{i+1}-m_{i}} f_{i}(R, N) N_{i}}_{\text {growing to class } i+1} \\
& -\underbrace{\mu_{i}^{e x t} N_{i}}_{\text {natural mortality }}-\underbrace{\mu_{i}(R, N) N_{i}}_{\text {cannibalism mortality }}
\end{aligned}
$$

And for the last class number $M$ :

$$
\dot{N}_{M}=\underbrace{\frac{k_{a}}{m_{M}-m_{M-1}} f_{M-1}(R, N) N_{M-1}}_{\text {arriving from class } M-1}-\underbrace{\mu_{M}^{e x t} N_{M}}_{\text {natural mortality }}-\underbrace{\mu_{M}(R, N) N_{M}}_{\text {cannibalism mortality }}
$$

For the individuals of the last weight class, assimilated biomass allows them to lay eggs. Only a fraction $\epsilon \in] 0,1]$ hatches and gives birth to individuals of 


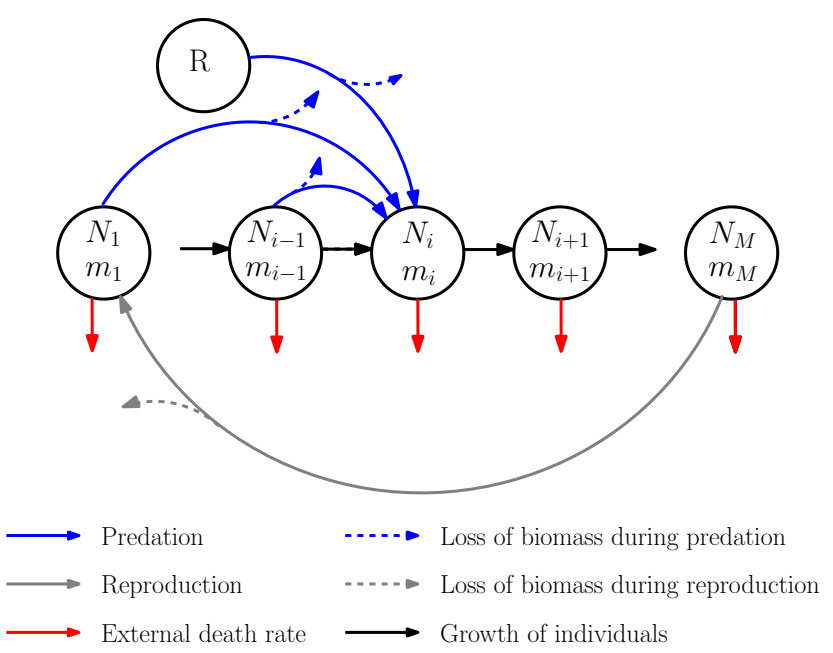

Fig. 1 Diagram of the modelled processes.

the first class so of weight $m_{1}$. Once again biomass and number individuals conservation give us:

$$
\begin{aligned}
\dot{N}_{1}= & \underbrace{\frac{\epsilon k_{a}}{m_{1}} f_{M}(R, N) N_{M}}_{\text {hatching eggs }}-\underbrace{\frac{k_{a}}{m_{2}-m_{1}} f_{1}(R, N) N_{1}}_{\text {growing to class } 2} \\
& -\underbrace{\mu_{1}^{\text {ext }} N_{1}}_{\text {natural mortality }}-\underbrace{\mu_{1}(R, N) N_{1}}_{\text {cannibalism mortality }}
\end{aligned}
$$

The idea of this article is to bring in light the impact of cannibalism within a population. Clearly, it is not attainable in a general case and we will restrict ourself to the study of a simple version with two weight classes (larvae and adults) that will allows us to go further in the mathematical study.

Remark 2 It's clear that we can go further in the modelling by including more biological processes. For example we can imagine that for some of the final classes (the mature ones) the assimilated biomass can be dispatched between growth and reproduction. Also we could have added a maintenance cost: a fixed quantity of energy (i.e. of biomass) may be paid by individuals for example to breath and to move. It would result in increased mortality rate when assimilated biomass can't allow individuals to pay this cost.

\subsection{A two weight classes model with cannibalism}

In this section, we will considerate a two weight classes model for the predators. The first one corresponding to larvae, the second one to adults. We will suppose 
that larvae feed only on the resource that allow them to become adults and adults spend a fraction of time $\kappa \in[0 ; 1]$ to feed on resource and a fraction $1-\kappa$ to feed on other adults, so there is only cannibalism within adults class. The assimilated biomass is then used to lay eggs. The model we will be interested in is the following one:

$$
\begin{aligned}
& \dot{R}=\Phi(R)-f_{1}(R) \cdot N_{1}-\kappa \cdot f_{2}(R) \cdot N_{2} \\
& \dot{N}_{1}=\alpha \cdot\left(\kappa \cdot f_{2}(R)+(1-\kappa) \cdot f_{c}\left(N_{2}\right)\right) \cdot N_{2}-\beta \cdot f_{1}(R) \cdot N_{1}-\mu_{1} \cdot N_{1} \\
& \dot{N}_{2}=\beta \cdot f_{1}(R) \cdot N_{1}-\mu_{2} \cdot N_{2}-\frac{1-\kappa}{m_{2}} f_{c}\left(N_{2}\right) \cdot N_{2}
\end{aligned}
$$

where we have introduced for simplification the constants:

$$
\alpha=\frac{\epsilon \cdot k_{a}}{m_{1}} \quad \text { and } \quad \beta=\frac{k_{a}}{m_{2}-m_{1}}
$$

We will assume also the following hypotheses on the functions :

(H7) The functions of predation $f_{*}(*=1,2, c)$ are strictly increasing and continuously differentiable on $\mathbb{R}^{+}$and verify $f_{*}(0)=0$.

(H8) The function $\Phi$ is continuously differentiable on $\left[0, R_{\max }\right]$, strictly positive on $] 0, R_{\max }$ [ and verify:

$$
\Phi(0)=\Phi\left(R_{\max }\right)=0, \quad \Phi^{\prime}(0)>0, \quad \Phi^{\prime}\left(R_{\max }\right)<0
$$

The initial condition is taken in the domain $\Omega=] 0, R_{\max }\left[\times \mathbb{R}_{+}^{*} \times \mathbb{R}_{+}^{*}\right.$. These hypotheses ensure, among other things, the existence and uniqueness of the solutions of the system.

The functional responses $f_{*}$ can be for example (and that's often the case) linear or functions with saturation. This second choice, probably more realistic from a biological point of view, will be done in section 4 . One of the most common growth function $\Phi$ is the logistic function (2.4) and we will also take this function for the study in section 4 .

$$
\Phi(R)=e R\left(1-\frac{R}{K_{c a p}}\right)
$$

where $e$ is the intrinsic growth rate of the resource and $K_{c a p}$ is the carrying capacity.

\section{Some general mathematical results}

In this section we will give some general results on the model defined in (2.2). Nevertheless the following proposition could be easily extended to the general model with $M$ classes. 
Proposition 1 Under the hypotheses $(H 7)$ and (H8), for all initial condition in $\Omega$, trajectories remain in a bounded set of $\Omega$ and we have:

$$
\limsup _{t \rightarrow \infty} N_{i}(t) \leq \frac{1}{m_{i}}\left(\frac{\sup _{\left[0 ; R_{\max }\right]} \Phi}{\min \left(\mu_{1}, \mu_{2}\right)}+R_{\max }\right)
$$

Proof We show that $\Omega$ is invariant. Considering the vector field at the boundaries of the domain, we obtain:

$$
\begin{array}{lll}
R \geq 0, N_{1}=0, N_{2} \geq 0 & \Longrightarrow & \dot{N}_{1}=\alpha \cdot\left(\kappa \cdot f_{2}(R)+(1-\kappa) \cdot f_{c}\left(N_{2}\right)\right) \cdot N_{2} \geq 0 \\
R \geq 0, N_{1} \geq 0, N_{2}=0 & \Longrightarrow & \dot{N}_{2}=\beta \cdot f_{1}(R) \cdot N_{1} \geq 0 \\
R=0, N_{1} \geq 0, N_{2} \geq 0 & \Longrightarrow & \dot{R}=0 \\
R=R_{\max }, N_{1} \geq 0, N_{2} \geq 0 & \Longrightarrow & \dot{R}=-f_{1}\left(R_{\max }\right) \cdot N_{1}-\kappa \cdot f_{2}\left(R_{\max }\right) \cdot N_{2} \leq 0
\end{array}
$$

To show that trajectories remain in a bounded set of $\Omega$, we consider the total biomass of the system at a time $t$ i.e. the following function:

$$
B(t)=R(t)+m_{1} N_{1}(t)+m_{2} N_{2}(t)
$$

The derivative of $B$ with respect to time is :

$$
\begin{aligned}
\dot{B}= & \Phi(R)-\left(1-k_{a}\right) f_{1}(R) N_{1}-\left(1-\epsilon k_{a}\right)\left(\kappa f_{2}(R)+(1-\kappa) f_{c}\left(N_{2}\right)\right) N_{2} \\
& -\mu_{1} m_{1} N_{1}-\mu_{2} m_{2} N_{2} \\
\leq & \Phi(R)-\min \left(\mu_{1}, \mu_{2}\right) B+\min \left(\mu_{1}, \mu_{2}\right) R \\
\leq & \sup _{\left[0 ; R_{\max }\right]} \Phi+\min \left(\mu_{1}, \mu_{2}\right) R_{\max }-\min \left(\mu_{1}, \mu_{2}\right) B
\end{aligned}
$$

And denoting $\mu_{m}=\min \left(\mu_{1}, \mu_{2}\right)$, we get:

$$
B(t) \leq B(0) e^{-\mu_{m} t}+\left(\frac{\sup _{\left[0 ; R_{\max }\right]} \Phi}{\mu_{m}}+R_{\max }\right)\left(1-e^{-\mu_{m} t}\right)
$$

From a biological point of view, this result is not surprising: it's clear that the only way the system can create biomass is with the growth of the resource. On another hand if $N_{1}$ or $N_{2}$ are too large, the linear mortality is sufficient to make the loss of biomass higher than the biomass created.

Introduce the following functions:

$$
\begin{gathered}
g(R, \kappa):=-\beta \kappa \alpha f_{1}(R) f_{2}(R)+\mu_{2}\left(\mu_{1}+\beta f_{1}(R)\right) \\
l(R):=\frac{\mu_{1}+\beta f_{1}(R)}{\beta f_{1}(R)}
\end{gathered}
$$


Proposition 2 The model (2.2) admits two trivial equilibria $E_{0 i}^{*}=(0,0,0)$ et $E_{0}^{*}=\left(R_{\max }, 0,0\right)$. The first one is always locally unstable, the second one is locally stable if and only if:

$$
g\left(R_{\max }, \kappa\right)>0
$$

Moreover if $E_{0}^{*}$ is locally stable, it is globally stable under one of the following sufficient conditions:

$$
\kappa f_{2}\left(R_{\max }\right) \geq \mu_{2} m_{2}
$$

or

$$
m_{2} \cdot \alpha<l\left(R_{\max }\right)
$$

Proof It's clear that under the hypotheses $(\mathrm{H} 7)$ and $(\mathrm{H} 8) E_{0 i}^{*}$ and $E_{0}^{*}$ are equilibria. To obtain the local stability results, we write the Jacobian matrix:

$$
J\left(E_{0 i}^{*}\right)=\left(\begin{array}{ccc}
\Phi^{\prime}(0) & 0 & 0 \\
0 & -\mu_{1} & 0 \\
0 & 0 & -\mu_{2}
\end{array}\right)
$$

and

$$
J\left(E_{0}^{*}\right)=\left(\begin{array}{ccc}
\Phi^{\prime}\left(R_{\max }\right) & -f_{1}\left(R_{\max }\right) & -\kappa f_{2}\left(R_{\max }\right) \\
0 & -\beta f_{1}\left(R_{\max }\right)-\mu_{1} & \kappa \alpha f_{2}\left(R_{\max }\right) \\
0 & \beta f_{1}\left(R_{\max }\right) & -\mu_{2}
\end{array}\right)
$$

Since $\Phi^{\prime}(0)>0, E_{0 i}^{*}$ is unstable with an instability direction in the positive octant because of its invariance. Concerning the Jacobian matrix of $E_{0}^{*}$, $\Phi^{\prime}\left(R_{\max }\right)<0$ is an eigenvalue, the two others are given by the eigenvalues of :

$$
\left(\begin{array}{cc}
-\beta f_{1}\left(R_{\max }\right)-\mu_{1} & \kappa \alpha f_{2}\left(R_{\max }\right) \\
\beta f_{1}\left(R_{\max }\right) & -\mu_{2}
\end{array}\right)
$$

Since the trace of this previous 2-dimensional matrix is negative, the stability of $E_{0}^{*}$ is given by the sign of the determinant of this matrix, (3.3) follows.

To obtain the global stability of $E_{0}^{*}$ results, let's remark first that if $N_{1}$ and $N_{2}$ tend to zero (extinction of the predators) then $R$ will go to $R_{\max }$.

Let's consider the Lyapunov function (3.6) defined for $\left(N_{1}, N_{2}\right) \in \mathbb{R}_{+}^{2}$. We will show that under some condition, $(0,0)$ is globally stable.

$$
V_{1}(t)=\mu_{2} N_{1}(t)+\kappa \alpha f_{2}\left(R_{\max }\right) \cdot N_{2}(t)
$$

The derivative of $V_{1}$ with respect to time is:

$$
\begin{aligned}
\dot{V}_{1}= & (1-\kappa) \cdot \alpha \cdot\left(\mu_{2}-\frac{\kappa}{m_{2}} f_{2}\left(R_{\max }\right)\right) f_{c}\left(N_{2}\right) \cdot N_{2} \\
& +\left(\beta f_{1}(R)\left(\kappa \alpha f_{2}\left(R_{\max }\right)-\mu_{2}\right)-\mu_{1} \mu_{2}\right) N_{1} \\
& +\kappa \alpha \mu_{2}\left(f_{2}(R)-f_{2}\left(R_{\max }\right)\right) N_{2}
\end{aligned}
$$


The first term (3.7) is negative if $\kappa f_{2}\left(R_{\max }\right) \geq \mu_{2} m_{2}$. The third term is strictly negative for $N_{2} \neq 0$ and the second one is strictly negative for $N_{1} \neq 0$ if $E_{0}^{*}$ is locally stable. So under the condition that $\kappa f_{2}\left(R_{\max }\right) \geq \mu_{2} m_{2}$ and $E_{0}^{*}$ is locally stable $E_{0}^{*}$ is globally stable.

Now we consider the following Lyapunov function:

$$
V_{2}(t)=N_{1}(t)+m_{2} \alpha N_{2}(t)
$$

The derivative of $V_{2}$ with respect to time is:

$$
\dot{V}_{2}(t)=\alpha \cdot\left(\kappa f_{2}(R)-\mu_{2} m_{2}\right) N_{2}+\left(\left(m_{2} \alpha-1\right) \beta f_{1}(R)-\mu_{1}\right) N_{1}
$$

The first term is strictly negative for $N_{2} \neq 0$ if $\kappa f_{2}\left(R_{\max }\right)<\mu_{2} m_{2}$. The second one is strictly negative for $N_{1} \neq 0$ if $m_{2} \alpha<l\left(R_{\max }\right)$. So under the condition that $\kappa f_{2}\left(R_{\max }\right)<\mu_{2} m_{2}$ and $m_{2} \alpha<l\left(R_{\max }\right), E_{0}^{*}$ is globally stable.

Combining the two last results we obtain (3.5).

Remark 3 Condition (3.3) shows that if individuals are highly cannibal (i.e. $\kappa$ is sufficiently close to 0$)$ then $E_{0}^{*}$ is locally stable. Numerical simulations show that in this case $E_{0}^{*}$ can be locally stable, but not globally stable and we can observe bi-stability even with simple functional responses like the linear ones. When individuals are not cannibal, if the trivial equilibrium is locally stable then it is globally stable since the first term of (3.7) becomes equal to zero when $\kappa=1$.

Proposition 3 There exists a positive non trivial equilibrium of the model for each solution on $] 0, R_{\max }$ [ of the following equation:

$$
g(R, \kappa)+h(R, \Psi(R, \kappa), \kappa)=0
$$

with:

$$
h\left(R, N_{2}, \kappa\right):=-\frac{1-\kappa}{m_{2}} f_{c}\left(N_{2}\right)\left(\beta f_{1}(R)\left(m_{2} \alpha-1\right)-\mu_{1}\right)
$$

and $\Psi$ defined on $] 0, R_{\max }\left[\right.$ as the unique $N_{2}>0$ verifying:

$$
s\left(R, N_{2}, \kappa\right):=\beta \Phi(R)-\left(\mu_{2}+\frac{1-\kappa}{m_{2}} f_{c}\left(N_{2}\right)+\kappa \beta f_{2}(R)\right) N_{2}=0
$$

Proof First observe that :

$$
\begin{gathered}
\dot{N}_{1}=0 \quad \Longleftrightarrow \quad N_{1}=N_{2} \alpha \frac{\kappa f_{2}(R)+(1-\kappa) f_{c}\left(N_{2}\right)}{\beta f_{1}(R)+\mu_{1}} \\
\dot{N}_{2}=0 \quad \Longleftrightarrow \quad N_{1}=N_{2} \frac{\mu_{2}+\frac{(1-\kappa)}{m_{2}} f_{c}\left(N_{2}\right)}{\beta f_{1}(R)}
\end{gathered}
$$

So there is a positive equilibrium if it exists $\left.\left(R, N_{2}\right) \in\right] 0, R_{\max }\left[\times \mathbb{R}_{+}^{*}\right.$ such that:

$$
g(R, \kappa)+h\left(R, N_{2}, \kappa\right)=0
$$


Moreover it requires:

$$
\dot{R}=0 \Longleftrightarrow s\left(R, N_{2}, \kappa\right)=0
$$

For $R$ fixed, $s$ is decreasing from $\beta \Phi(R)>0$ to $-\infty$ when $N_{2}$ varies from 0 to $+\infty$, the uniqueness of $\Psi(R, \kappa)$ follows.

For this general model, obtaining more precise global results becomes intricate. Nevertheless simulations show that cannibalism may have a stabilizing role (as we can see on Fig. 2). In the following section, we will simplify further the model with hypotheses on the differences between the time scales of the resource and the predator to obtain more precise results.
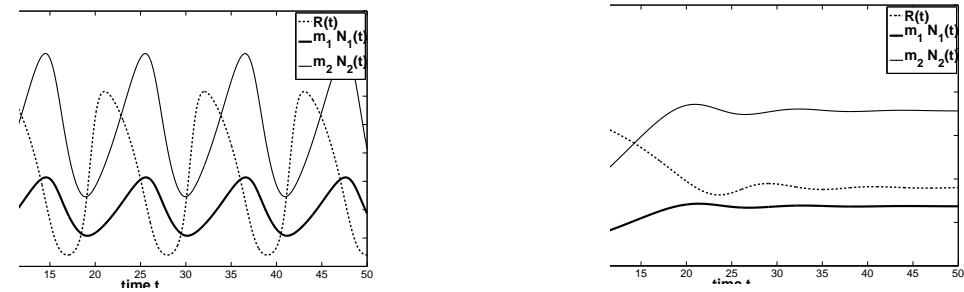

Fig. 2 On the left figure there is no cannibalism $(\kappa=1)$ and we can observe oscillations. On the right figure $\kappa=0.7$, other parameters are unchanged and there is convergence to a positive equilibrium.

\section{Slow-fast dynamics}

\subsection{Hypotheses}

In this section, we will show that cannibalism can stabilize a positive equilibrium. For that, we will suppose that the resource has a fast dynamics compared to predator dynamics and we will study the limiting slow-fast system. Such hypothesis is quite classical in biology (see e.g. (Rinaldi and Muratori, 1992)). The functional responses we will use are the following ones:

$$
f_{i}(R)=d_{i} \frac{R}{R_{h}+R} \quad \text { and } \quad f_{c}\left(N_{2}\right)=d_{2} \cdot \frac{N_{2}}{N_{h}+N_{2}}
$$

The growth function of the resource will be taken logistic:

$$
\Phi(R)=e R\left(1-\frac{R}{K_{c a p}}\right)
$$

The coefficients $d_{i}$ (in biomass per unit of time) correspond to the maximum ingestion rate. The choice of the same half saturation constant for larvae and adults means that the ratio between the maximum ingestion rate and the attack rate is the same for both of them. 
Remark 4 If moreover we suppose that it is harder for an adult to eat themselves than to eat the resource, it is suitable to take $m_{2} N_{h}>R_{h}$ : that means that the attack rate on adults is less important than the attack rate on the resource.

In order to be in a slow-fast system framework, we attribute to parameters different orders of magnitude. We will assume that $e \gg \mu_{1}$ to fix the two time scales; then we have to choose $d_{1} / e$ et $d_{2} / e$ appreciable (neither very small nor very large) and also $\mu_{2} / \mu_{1}$ appreciable (to ensure that there is not two time scales within the predator population) and finally $\alpha d_{2} / \mu_{1}$ and $\beta d_{1} / \mu_{2}$ also appreciable to get the two time scales: fast resource dynamics compared to the larvae and adult dynamics. To get the suitable order of magnitude for $\alpha, \beta$, we can adjust the parameters $k_{a}, \epsilon, m_{1}$ and $m_{2}$. We will also assume that $\kappa>0$ and make the hypothesis (H9) that allows to be in an interesting situation for the study.

(H9) $R_{h}<K_{\text {cap }}$

4.2 Study of the limiting slow-fast system

The slow manifold is defined by $\dot{R}=0$ i.e.:

$$
R .\left(R^{2}+\left(R_{h}-K_{c a p}\right) R+\frac{K_{c a p}}{e}\left(d_{1} N_{1}+\kappa \cdot d_{2} N_{2}-e R_{h}\right)\right)=0
$$

Under the hypothesis (H9) this manifold is made of three sub-manifolds in the positive octant (see Fig. 3 for a graphical representation) that will be parametrized with $N_{1}, N_{2}$ as following:

$$
\begin{aligned}
& S^{0}=\left\{\left(N_{1}, N_{2}, 0\right):\left(N_{1}, N_{2}\right) \in \mathbb{R}_{+}^{2}\right\} \\
& S_{\kappa}^{+}=\left\{\left(N_{1}, N_{2}, s_{+}\left(N_{1}, N_{2}, \kappa\right)\right):\left(N_{1}, N_{2}\right) \in \Omega_{\kappa}^{+}\right\} \\
& S_{\kappa}^{-}=\left\{\left(N_{1}, N_{2}, s_{-}\left(N_{1}, N_{2}, \kappa\right)\right):\left(N_{1}, N_{2}\right) \in \Omega_{\kappa}^{-}\right\}
\end{aligned}
$$

with the domains of definition:

$$
\begin{aligned}
& \Omega_{\kappa}^{+}=\left\{\left(N_{1}, N_{2}\right) \in \mathbb{R}_{+}^{2}: \frac{d_{1}}{e} N_{1}+\kappa \frac{d_{2}}{e} N_{2} \leq \frac{1}{4 K_{\text {cap }}}\left(K_{\text {cap }}+R_{h}\right)^{2}\right\} \\
& \Sigma_{\kappa}=\left\{\left(N_{1}, N_{2}\right) \in \mathbb{R}_{+}^{2}: \frac{d_{1}}{e} N_{1}+\kappa \frac{d_{2}}{e} N_{2} \leq R_{h}\right\} \\
& \Omega_{\kappa}^{-}=\overline{\Omega_{\kappa}^{+} \backslash \Sigma_{\kappa}}
\end{aligned}
$$

and with:

$$
\begin{aligned}
& s_{ \pm}\left(N_{1}, N_{2}, \kappa\right)=\frac{1}{2}\left(K_{c a p}-R_{h} \pm \sqrt{\Delta\left(N_{1}, N_{2}, \kappa\right)}\right) \\
& \Delta\left(N_{1}, N_{2}, \kappa\right)=\left(K_{c a p}+R_{h}\right)^{2}-4 \frac{K_{c a p}}{e}\left(d_{1} N_{1}+\kappa d_{2} N_{2}\right)
\end{aligned}
$$


The fold of this slow manifold is the intersection between the manifold $S_{\kappa}^{+}$ et $S_{\kappa}^{-}$. The value of $R$ on the fold is independent of $N_{1}, N_{2}$ and is equal to $\left(K_{\text {cap }}-R_{h}\right) / 2$. The projection of this fold on the plane $R=0$ has for equation:

$$
\delta_{\kappa}^{S}=\left\{\left(N_{1}, N_{2}\right) \in \mathbb{R}_{+}^{2}: \frac{d_{1}}{e} N_{1}+\kappa \frac{d_{2}}{e} N_{2}=\frac{1}{4 K_{\text {cap }}}\left(K_{\text {cap }}+R_{h}\right)^{2}\right\}
$$

The intersection between $S_{\kappa}^{-}$and the manifold $S_{0}$ is the set of $\left(N_{1}, N_{2}, 0\right)$ such that $\left(N_{1}, N_{2}\right) \in \delta_{\kappa}^{0}$ where:

$$
\delta_{\kappa}^{0}=\left\{\left(N_{1}, N_{2}\right) \in \mathbb{R}_{+}^{2}: \frac{d_{1}}{e} N_{1}+\kappa \frac{d_{2}}{e} N_{2}=R_{h}\right\}
$$

Remark 5 It is clear that $\Sigma_{\kappa} \subset \Omega_{\kappa}^{+}$.

Proposition 4 We have:

- $S_{\kappa}^{-}$is repulsive $\forall\left(N_{1}, N_{2}\right) \in \operatorname{int}\left(\Omega_{\kappa}^{-}\right)$.

- $S_{\kappa}^{+}$is attractive $\forall\left(N_{1}, N_{2}\right) \in \operatorname{int}\left(\Omega_{\kappa}^{+}\right)$.

- $S^{0}$ is repulsive $\forall\left(N_{1}, N_{2}\right) \in \operatorname{int}\left(\Sigma_{\kappa}\right)$ and attractive $\forall\left(N_{1}, N_{2}\right) \in \operatorname{int}\left(\mathbb{R}_{+}^{2} \backslash \Sigma_{\kappa}\right)$

- The boundary between the basin of attraction of $S^{0}$ and $S_{\kappa}^{+}$for $\left(N_{1}, N_{2}\right) \in$ $\operatorname{int}\left(\Omega_{\kappa}^{-}\right)$is $S_{\kappa}^{-}$.

Proof This previous proposition is an immediate consequence of the study of the sign of $\dot{R}$ in the different domains of the positive octant.

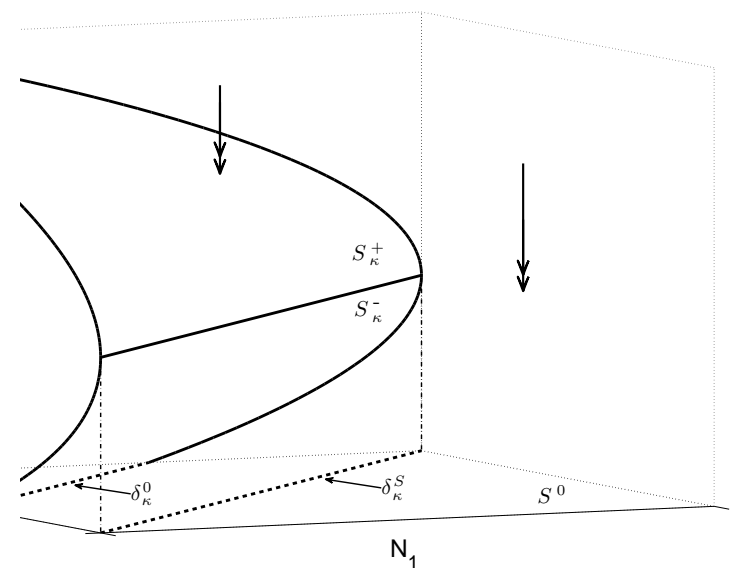

Fig. 3 Shape of the manifolds when $0<R_{h}<K_{\text {cap }}$. The double arrows correspond to the direction of the fast dynamic

Now we will study the slow system on the slow manifolds. For that we will choose $R_{h}>0$ non infinitesimal (which means that $R_{h}$ is not in a very small 
neighbourhood of 0$)$. Then $f_{i}(0)=0$, and the slow system is globally defined with a parameter $R_{h}$. In the slow system, there is no more infinitesimals, and we can consider that the case $R_{h}>0$ is a regular perturbation of the case $R_{h}=0$, at least for $R$ bigger than some constant. We then prove by structural stability, some generic results valid for $R_{h}$ non infinitesimal but smaller than some constant. For notions regarding regular perturbations and structural stability see (Hirsch and Smale, 1974).

Proposition $5(0,0)$ is the global attractor of the slow dynamics on $S_{0}$.

Proof The equations of the field on $S_{0}$ are exactly:

$$
\begin{aligned}
& \dot{N}_{1}=-\mu_{1} N_{1}+\alpha(1-\kappa) f_{c}\left(N_{2}\right) N_{2} \\
& \dot{N}_{2}=-\mu_{2} N_{2}-\frac{1}{m_{2}}(1-\kappa) f_{c}\left(N_{2}\right) N_{2}
\end{aligned}
$$

The equation $\dot{N}_{2}$ implies that $N_{2}$ goes to 0 and so $N_{1}$ goes to 0 too. From a biological point of view, it is clear that if there is not any external supply of biomass for the predator $(R=0$ so no supply of biomass when feeding on the resource), and because of the fixed linear death rate, the predator population goes to extinction. The adult cannibalism can have only a positive impact on the extinction, since the efficiency of biomass conversion $k_{a}$ is less than 1 .

Corollary 1 Consequently for each initial condition on $S_{0}$, trajectories eventually enter $\operatorname{int}\left(\Sigma_{\kappa}\right)$ where this manifold is repulsive and exit it after some delay. Hence we can defined the input-output map $\Psi_{0}$ (continuous if $R_{h}$ is sufficiently small) which associate to an entrance point $\left(N_{1}^{0}, N_{2}^{0}\right) \in \mathbb{R}_{+}^{2} \backslash \Sigma_{\kappa}$ an exit point $\left(N_{1}\left(t_{0}\right), N_{2}\left(t_{0}\right)\right) \in \Sigma_{\kappa}$ with $t_{0}>0$ such as:

$$
\int_{0}^{t_{0}}\left(e-\frac{d_{1}}{R_{h}} N_{1}(t)-\kappa \frac{d_{2}}{R_{h}} N_{2}(t)\right) d t=0
$$

Where $\left(N_{1}(0), N_{2}(0)\right)=\left(N_{1}^{0}, N_{2}^{0}\right)$ and $\left(N_{1}, N_{2}\right)$ follows the dynamics (4.3) of the field on $S_{0}$.

The continuity of this map for $R_{h}$ sufficiently small is a consequence of the transversality of the field on $\delta_{\kappa}^{0}$. Indeed we have:

$$
\frac{d_{1}}{e} \dot{N}_{1}+\kappa \frac{d_{2}}{e} \dot{N}_{2}=-\frac{d_{1} \mu_{1}}{e} N_{1}+\left(-\frac{\kappa d_{2} \mu_{2}}{e}+(1-\kappa)\left(\frac{\alpha d_{1}}{e}-\frac{\kappa d_{2}}{m_{2} e}\right) f_{c}\left(N_{2}\right)\right) N_{2}
$$

If $R_{h}$ is sufficiently small, the values taken by $N_{2}$ on $\delta_{\kappa}^{0}$ are small enough (see the expression (4.2)) and so $f_{c}\left(N_{2}\right)$ is sufficiently close to 0 to ensure that this previous expression is always negative. 
4.3 Model without cannibalism

We will now study the model without cannibalism, i.e. when $\kappa=1$ and show that, under some conditions, the limiting model does not have any stable positive equilibrium and trajectories converge to a relaxation cycle.

Proposition 6 If $\mu_{2}\left(\mu_{1}+\beta d_{1}\right)-\alpha d_{2} \beta d_{1}>0$, then for $R_{h}$ sufficiently small, $(0,0)$ is the global attractor of the slow field on $S_{1}^{+}$.

If $\mu_{2}\left(\mu_{1}+\beta d_{1}\right)-\alpha d_{2} \beta d_{1}<0$, then for $R_{h}$ sufficiently small, every trajectory leaves the manifold $S_{1}^{+}$through the fold.

If moreover $\beta d_{2}>\mu_{1}+\beta d_{1}$ and $\alpha d_{1}>\mu_{2}$, then for $R_{h}$ sufficiently small, trajectories leave the manifold transversally to the fold.

Proof The slow field on $S_{1}^{+}$has for equation:

$$
\left(\begin{array}{c}
\dot{N}_{1} \\
\dot{N}_{2}
\end{array}\right)=\left(\begin{array}{cc}
-\mu_{1}-\beta f_{1}\left(s_{+}\left(N_{1}, N_{2}\right)\right) & \alpha f_{2}\left(s_{+}\left(N_{1}, N_{2}\right)\right) \\
\beta f_{1}\left(s_{+}\left(N_{1}, N_{2}\right)\right) & -\mu_{2}
\end{array}\right)\left(\begin{array}{c}
N_{1} \\
N_{2}
\end{array}\right)
$$

Taking into account that $R_{h}<K_{\text {cap }}$, we have for each $N_{1}, N_{2}$ :

$$
s_{+}\left(N_{1}, N_{2}\right) \geq \frac{1}{2}\left(K_{\text {cap }}-R_{h}\right)
$$

Consequently:

$$
f_{i}\left(s_{+}\left(N_{1}, N_{2}\right)\right)=d_{i} \frac{s_{+}\left(N_{1}, N_{2}\right)}{R_{h}+s_{+}\left(N_{1}, N_{2}\right)} \in\left[d_{i} \frac{K_{c a p}-R_{h}}{K_{c a p}+R h}, d_{i}\right] \quad i=1,2
$$

Now we will study the slow field on $S_{1}^{+}$considering $R_{h}$ as a parameter. In the case of $R_{h}=0$, this slow field is linear and satisfies:

$$
\left(\begin{array}{c}
\dot{N}_{1} \\
\dot{N}_{2}
\end{array}\right)=\left(\begin{array}{cc}
-\mu_{1}-\beta d_{1} & \alpha d_{2} \\
\beta d_{1} & -\mu_{2}
\end{array}\right)\left(\begin{array}{c}
N_{1} \\
N_{2}
\end{array}\right)
$$

The trace of this linear system is negative and its determinant is given by:

$$
\mu_{2}\left(\mu_{1}+\beta d_{1}\right)-\alpha d_{2} \beta d_{1}
$$

If this determinant is strictly positive, then $(0,0)$ is globally stable. In particular every compact of $\mathbb{R}_{+}^{2}$ is included in the basin of attraction of the null equilibrium.

If this determinant is strictly negative, the $(0,0)$ is a saddle point. Its unstable variety is in the positive quadrant and the stable one is not in the positive quadrant. This is an immediate consequence of the invariance of $\mathbb{R}_{+}^{2}$. Consequently, every trajectory goes to infinity and so leaves out any compact of $\mathbb{R}_{+}^{2}$. The trajectories will leave out the domain transversally to the fold if the following quantity is positive for $\left(N_{1}, N_{2}\right) \in \delta_{1}^{S}$ :

$$
\frac{d_{1}}{e} \dot{N}_{1}+\frac{d_{2}}{e} \dot{N}_{2}=\frac{d_{1}}{e}\left(-\mu_{1}-\beta d_{1}+\beta d_{2}\right) N_{1}+\frac{d_{2}}{e}\left(\alpha d_{1}-\mu_{2}\right) N_{2}
$$


The hypotheses done in the third part of proposition 6 ensure that this quantity is always positive.

This properties being structurally stable, if $R_{h}$ is sufficiently small they are still verified.

Theorem 1 Assume that $\beta d_{2}>\mu_{1}+\beta d_{1}$ and $\alpha d_{1}>\mu_{2}$. If $R_{h}$ is sufficiently small there exists at least one relaxation cycle for the limiting slow-fast system in the interior of the positive octant.

Proof To establish the existence of a such relaxation cycle, we will consider the first return map from the fold to itself. More precisely we will consider the projection on $S^{0}$ of this map i.e. the map defined on $\delta_{1}^{S}$. We can distinguish four phases:

- The fast fall from the fold on the manifold $S^{0}$. In the limiting system $N_{1}$ and $N_{2}$ do not change.

- The trajectory goes along this manifold and ends to leave it. The entry-exit map $\Psi_{0}$ has been defined in the corollary 1 .

- The fast rise from $S^{0}$ to $S_{1}^{+}$. Once again, in the limiting system $N_{1}$ and $N_{2}$ do not change.

- The escape from the manifold $S_{1}^{+}$through the fold. We denote $\Psi_{S}$ the continuous map (because of the transversality of the field on the fold) which associates to a point in $\Omega_{1}^{+} \backslash(0,0)$ the exit point of $\delta_{1}^{S}$.

The first return map is given by $\Psi_{S} \circ \Psi_{0}$. This application from $\delta_{1}^{S}$ to itself is continuous and so admit at least one fixed point. Moreover, because of the repulsivity of the slow field on the boundaries $N 1=0$ and $N_{2}=0$ of $S_{1}^{+}$, we have $\Psi_{S}\left(\delta_{1}^{0}\right) \subset \operatorname{int}\left(\delta_{1}^{S}\right)$. Consequently the image of $\delta_{1}^{S}$ by $\Psi_{S} \circ \Psi_{0}$ is in the interior of $\delta_{1}^{S}$ and so every fixed point of this application is in the interior of $\delta_{1}^{S}$

We proved that there exists at least one relaxation cycle for the limiting system. We can not conclude on the existence of a such limit cycle in the slow-fast system, nevertheless trajectories remains close to a relaxation cycle obtained in the limiting system.

Numerical simulations seem to show that there is a relaxation cycle in the slow-fast system which is unique and globally attractive. See Fig. 5 and Fig. 6 for a numerical illustration.

\subsection{Add of cannibalism}

We will now add some cannibalism, i.e. take values of $\kappa$ different than 1 . Moreover we will consider the case where the trivial equilibrium without cannibalism is unstable. Consequently we have $\underline{\kappa}<1$ with:

$$
\underline{\kappa}=\frac{\mu_{2}}{\alpha d_{2}} \frac{\mu_{1}+\beta d_{1}}{\beta d_{1}}
$$


Proposition 7 (case $R_{h}=0$ )

Assume that $\underline{\kappa}>m_{2} \mu_{2} / d_{2}$, then there exists $\bar{\kappa}>\underline{\kappa}$ such that for all $\left.\kappa \in\right] \underline{\kappa}, \bar{\kappa}[$, there exists a unique positive equilibrium on $S_{\kappa}^{+}$.

Proof To establish this result, we will study the intersection of the nullclines in the domain $\operatorname{int}\left(\Omega_{\kappa}^{+}\right)$. For $R_{h}=0$, the slow system on $S_{\kappa}^{+}$is:

$$
\begin{aligned}
& \dot{N}_{1}=\alpha\left(\kappa d_{2}+(1-\kappa) f_{c}\left(N_{2}\right)\right) N_{2}-\beta d_{1} N_{1}-\mu_{1} N_{1} \\
& \dot{N}_{2}=\beta d_{1} N_{1}-\mu_{2} N_{2}-\frac{1-\kappa}{m_{2}} f_{c}\left(N_{2}\right) N_{2}
\end{aligned}
$$

Then we can write the equation of the nullclines $\dot{N}_{1}=0$ and $\dot{N}_{2}=0$ :

$$
\begin{aligned}
& N_{1}=\left(\frac{\alpha \kappa d_{2}}{\mu_{1}+\beta d_{1}}\right) N_{2}+\left(\frac{\alpha(1-\kappa)}{\mu_{1}+\beta d_{1}}\right) f_{c}\left(N_{2}\right) N_{2}:=g_{1}\left(N_{2}\right) \\
& N_{1}=\left(\frac{\mu_{2}}{\beta d_{1}}\right) N_{2}+\left(\frac{(1-\kappa)}{m_{2} \beta d_{1}}\right) f_{c}\left(N_{2}\right) N_{2}:=g_{2}\left(N_{2}\right)
\end{aligned}
$$

These two nullclines intersect (not necessary in $\operatorname{int}\left(\Omega_{\kappa}^{+}\right)$) when $g_{1}\left(N_{2}\right)-$ $g_{2}\left(N_{2}\right)=0$, which gives:

$$
\underbrace{\left(\frac{\kappa}{\underline{\kappa}}-1\right)}_{a(\kappa)}+\underbrace{(1-\kappa)\left(\frac{1}{\underline{\kappa}}-\frac{d_{2}}{m_{2} \mu_{2}}\right)}_{b(\kappa)} \frac{f_{c}\left(N_{2}\right)}{d_{2}}=0
$$

For $\kappa>\underline{\kappa}$ and under the assumption that $\underline{\kappa}>m_{2} \mu_{2} / d_{2}$ we have $a(\kappa)>0$ et $b(\kappa)<0$. So $(4.5)$ admits at most one solution. Moreover:

$$
\lim _{\kappa \downarrow \underline{\kappa}} a(\kappa)=0 \quad \text { and } \quad \lim _{\kappa \downarrow \underline{\kappa}} b(\kappa) \neq 0
$$

Which yield, taking into account that $f_{c}(0)=0$, that the solution $N_{2}^{*}(\kappa)$ of (4.5) tends to 0 when $\kappa$ decrease to $\underline{\kappa}$. Consequently $N_{1}^{*}(\kappa)=g_{1}\left(N_{2}^{*}(\kappa)\right)$ also tends to 0 . Finally, due to the continuity of the equilibrium value with respect to $\kappa$, there exists $\bar{\kappa}>\kappa$ such that:

$$
\forall \kappa \in] \underline{\kappa}, \bar{\kappa}\left[, 0<\frac{d_{1}}{e} N_{1}^{*}(\kappa)+\kappa \frac{d_{2}}{e} N_{2}^{*}(\kappa)<\frac{K_{c a p}}{4}\right.
$$

Proposition 8 (Case $R_{h} \neq 0$ )

If $\underline{\kappa}>m_{2} \mu_{2} / d_{2}$ and $R_{h}$ is sufficiently small, then there exists an interval included in $] \underline{\kappa}, 1$ [ such that the model has a unique positive equilibrium on $S_{\kappa}^{+}$.

Proof When $R_{h}=0$, there is an unique solution of $g_{1}\left(N_{2}\right)-g_{2}\left(N_{2}\right)=0$. It is easy to see that the derivative of this function is strictly positive. This result is structurally stable and then true for $R_{h}$ sufficiently small. The claim follows. 
Theorem 2 Assume that $\underline{\kappa}>m_{2} \mu_{2} / d_{2}$ and $R_{h}$ sufficiently small and the existence of a positive equilibrium on $S_{\kappa}^{+}$, then there exists $\left(\bar{N}_{1}, \bar{N}_{2}\right) \in \delta_{\kappa}^{S}$ such that the domain $V$, except a small neighbourhood of $S_{\kappa}^{-}$, is included in the basin of attraction of this positive equilibrium.

$$
\left.\left.\left.\left.V=\left[0, \bar{N}_{1}\right] \times\left[0, \bar{N}_{2}\right] \times\right] 0, K_{\text {cap }}\right] \backslash\{(0,0, R): R \in] 0, K_{\text {cap }}\right]\right\}
$$

Lemma 1 Under the condition of theorem 2, there exists $\left(\bar{N}_{1}, \bar{N}_{2}\right) \in \delta_{\kappa}^{S}$ such that the domain $D \subset S_{\kappa}^{+}$defined in (4.6) verify:

- The positive equilibrium is in $D$

- $\Sigma_{\kappa} \subset \tilde{D}$

- $D$ is invariant by the slow field of $S_{\kappa}^{+}$ $D=\left\{\left(N_{1}, N_{2}, s_{+}\left(N_{1}, N_{2}, \kappa\right)\right):\left(N_{1}, N_{2}\right) \in \tilde{D}:=\left[0, \bar{N}_{1}\right] \times\left[0, \bar{N}_{2}\right]\right\}$

Proof For $R_{h}=0$, the study of the slow field directions on $S_{\kappa}^{+}$allows to show the existence of $\left(\bar{N}_{1}, \bar{N}_{2}\right) \in \delta_{\kappa}^{S}$ such that the slow field is transversal to the fold oriented toward the interior of $S_{\kappa}^{+}$(see Fig. 4 for a graphical illustration). Moreover on the boundary of $D$, the slow field is oriented towards the interior (except for $\left(0,0, K_{\text {cap }}\right)$ where it is equal to 0 ) and for $R_{h}=0, \Sigma_{\kappa}$ is reduced to the point $(0,0)$ which is included in $\tilde{D}$.

For $R_{h}$ sufficiently small, the same domain $D$ verifies the three conditions. The equilibrium was in the interior of $D$ for $R_{h}=0$ so this is still the case for $R_{h}$ sufficiently small. Moreover the field was oriented towards the interior and the domain $\Sigma_{\kappa}$ increases continuously with $R_{h}$, so once again the two last conditions are verified for $R_{h}$ sufficiently small.

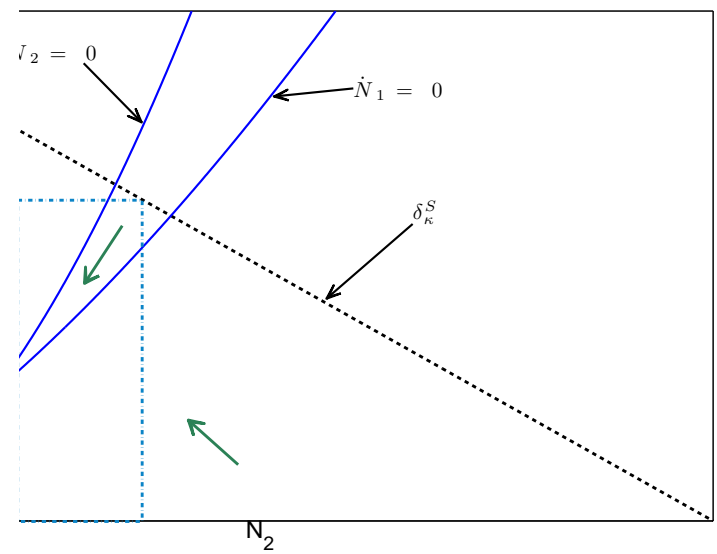

Fig. 4 Projection on the plane $\left(N_{1}, N_{2}\right)$ of the nullclines of the slow field on $S^{+}$. The green arrows correspond to the direction of the slow field. 
Proof (Theorem 2) In order to show that all trajectories in $V$ go to the positive equilibrium, we will firstly show that this positive equilibrium is the global attractor of the slow field for every trajectory starting in $D$. For $R_{h}=0$, we can write the Jacobian matrix of the slow system on $S_{\kappa}^{+}$as following:

$$
J_{\kappa}\left(N_{1}, N_{2}\right)=\left(\begin{array}{cc}
-\mu_{1}-\beta d_{1} & \alpha d_{2} \kappa+\alpha d_{2}(1-\kappa)\left(1-\frac{N_{h}^{2}}{\left(N_{h}+N_{2}\right)^{2}}\right) \\
\beta d_{1} & -\mu_{2}-d_{2} \frac{(1-\kappa)}{m_{2}}\left(1-\frac{N_{h}^{2}}{\left(N_{h}+N_{2}\right)^{2}}\right)
\end{array}\right)
$$

The trace of this Jacobian matrix always being negative and since we are in dimension 2 and $D$ is invariant by the slow field, we obtain by the Dulac criterion that there cannot be any limit cycle in $D$. Trajectories starting in $D$ goes to an equilibrium in $D$, the trivial equilibrium or the positive one. Since the trivial one is unstable, the trajectories converge toward the positive one (unless they start exactly at the trivial equilibrium).

Once again, if $R_{h}$ is sufficiently small, this property is verified and the positive equilibrium is a global attractor of the slow field on $D \backslash\left(0,0, K_{\text {cap }}\right)$.

The study of the fast dynamics in the volume $V$ allows us to conclude. Two cases of figure can occur: either the trajectory starts in the part of $V$ where $S_{\kappa}^{+}$is attractive, then the trajectory arrives in $D$ and goes to the positive equilibrium. Or the trajectory starts in the part of $V$ where $S^{0}$ is attractive, leaves out this manifold when $\left(N_{1}, N_{2}\right) \in \Sigma_{\kappa} \subset \tilde{D}$ and then goes to $D$ and eventually converges to the positive equilibrium.

Remark 6 It's clear that $V$ is the basin of attraction for many trajectories. Every trajectory that enters in a small neighbourhood of $S^{0}$ will eventually enter the domain $V$ (they will leave that small neighbourhood in $\Sigma_{\kappa}$ ). For examples, trajectories that fall down on $S^{0}$ by the fold or trajectories that fall down from $S_{\kappa}^{-}$. Moreover for the slow-field on $S_{\kappa}^{+}$the basin of attraction of the positive equilibrium is not restricted to $D$ so every trajectory that enters this basin will also converge to the positive equilibrium.

\section{Numerical simulation}

In order to illustrate results obtained in the previous section, we show some simulations. Parameters used are $e=10, \mu_{1}=1, d_{1}=10, d_{2}=30, \mu_{2}=1$, $K_{\text {cap }}=1, m_{1}=1, m_{2}=11, k_{a}=0.8, \epsilon=0.2, N_{h}=1$ and $R_{h}=0.1$. The difference of scale between the dynamic of $R$ and the dynamics of $N_{1}$ and $N_{2}$ is then about 10. To this set of parameters are associated the values $\alpha=0.16$ and $\beta=0.08$ (see (2.3) for the expressions) and $\underline{\kappa} \approx 0.47$

This set of parameters also ensures that the conditions of theorem 1 are satisfied $\left(\beta d_{2}>\mu_{1}+\beta d_{1}\right.$ and $\left.\alpha d_{1}>\mu_{2}\right)$ so in the limiting system, there is a relaxation cycle without cannibalism and we can see that it is also the case for the chosen parameters on the left figure of Fig. 5 and Fig. 6. Moreover the condition of theorem 2 is also verified $\left(\underline{\kappa}>m_{2} \mu_{2} / d_{2}\right)$ and so, for some levels 
of cannibalism the positive equilibrium is stable.

In Fig. 7, we show a bifurcation diagram; we plotted the asymptotic behaviour of the model when $\kappa$ vary from 0.3 (high level of cannibalism) to 1 (no cannibalism). The y-axis corresponds to the period of oscillations. So if it is equal to zero, there is convergence of the system, otherwise there is a limit cycle. the Hopf bifurcation appears when a stable equilibrium gives birth to a limit cycle, here for $\kappa \approx 0.65$.
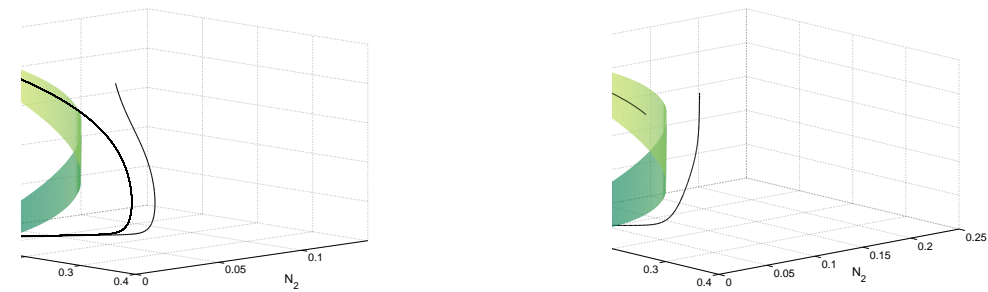

Fig. 5 On the left figure $\kappa=1$, the trajectory converges to a relaxation cycle. On the right figure $\kappa=0.6$, there is convergence to a positive equilibrium on $S_{\kappa}^{+}$. The slow manifolds $S_{\kappa}^{-}$ and $S_{\kappa}^{+}$are plotted in green.
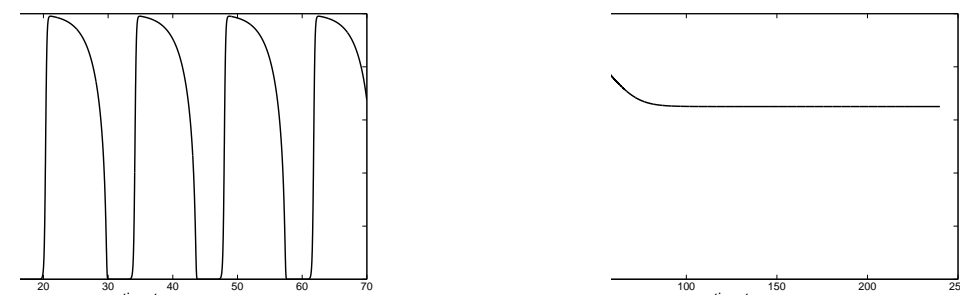

Fig. 6 Evolution of the resource $R$. On the left $\kappa=1$ : We can see the alternation between slow and fast dynamics. On the right $\kappa=0.6$, after having reached the manifold $S_{\kappa}^{+}$, there is convergence to the equilibrium. 


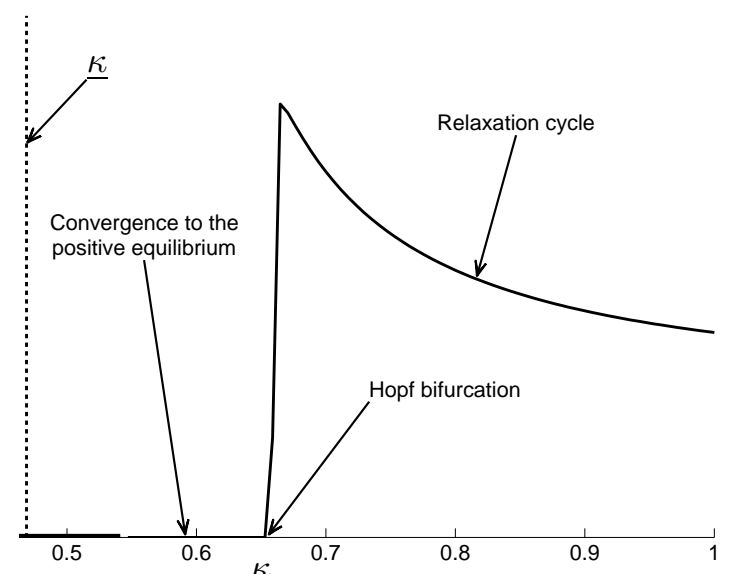

Fig. 7 Asymptotic behaviour of the system when $\kappa$ vary from 0.3 to 1 : If individuals are too cannibal $(\kappa \geqq 0.54)$, there is extinction of the predators, then for $0.54 \lesssim \kappa \lesssim 0.65$, there is convergence to a positive equilibrium on $S_{\kappa}^{+}$which losses its stability to give birth to relaxation limit cycle when $0.65 \lesssim \kappa$.

\section{Discussion and Conclusions}

The first contribution of this paper is a new discrete weight/stage structured prey-predator model including predation on a prey but also cannibalism on other predator weight classes enabling individuals to pass from a class to the following one. This model can be seen as a discretization in weight of continuous weight-structured PDE models (see (Benoît and Rochet, 2004; Maury et al, 2007)). Moreover this model conserves number of individuals and biomass so it can be easily integrated in more detailed ecological models where describing biomass fluxes is important like N.P.Z. models used in the study of planktonic ecosystem (see e.g. (Riley, 1946)).

It has been shown in this article that under some hypotheses, cannibalism may stabilize a weight-structured system. One of the main assumption done is that resource has a fast dynamic compared to the predator's one. Indeed it can be criticized: it is not sure that it is biologically realistic, especially because it leads to relaxation cycle where resource regularly takes values very closed to zero. In simulations of section 5 for a ratio of 10 between the dynamics of the prey and the dynamics of the predator, the relative variation of the biomass of prey during a relaxation cycle is about $10^{9}$ : the validity of the model in such extreme cases is not so clear.

Moreover numerical simulations seem to show that destabilisation of an equilibrium when adding cannibalism never appears in this model contrary to (Magnússon, 1999). But fundamentally the problem is here quite different since the cannibalism occurs within the adult population whereas in (Magnússon, 
1999) cannibalism means feeding on larvae by adults. Moreover some computations (not shown in this article) proved that it is also possible to obtain a locally stable positive equilibrium for all values of $\kappa$. In that case, the stabilization aspect of cannibalism is not so relevant. But there are other questions to wonder about cannibalism such as: can cannibalism maximise some criterion like the total biomass? Another question is related to evolution: for example, can cannibalism allows a population not to be invaded by an other more or less cannibalistic population? Once again simulations seem to answer positively these two questions, which are the subject of a current work.

In section 4 , we have supposed that the trivial equilibrium was unstable and studied the model for level of cannibalism for which it is the case. As we said in remark 3 , if the predators are cannibal above some threshold then the trivial equilibrium is locally stable, but it doesn't mean that it is globally stable. Once again in numerical simulations we have observed bi-stability. In some way it can be dangerous for the ecosystem: we have to keep in mind that environment (temperature, but also other species or even humans) has an important role in the dynamics and if for some reason a perturbation occurs and leads the ecosystem in the basin of attraction of the trivial equilibrium, it may have for consequence the extinction of that species.

\section{References}

Benoît E (1983) Systèmes lents-rapides dans $\mathbb{R}^{3}$ et leurs canards. In: Troisième rencontre du Schnepfenried, Société mathématique de France, pp 159-191, astérisque volume 109-110(2)

Benoît E, Rochet M (2004) A continuous model of biomass size spectra governed by predation and the effects of fishing on them. Journal of Theoretical Biology 226(1):9-21

Birkhead T, Lee K, Young P (1988) Sexual cannibalism in the praying mantis hierodula membranacea. Behaviour 106(1-2):112-118

Claessen D, de Roos A, Persson L (2004) Population dynamic theory of sizedependent cannibalism. Proceedings of the Royal Society of London Series B: Biological Sciences 271(1537):333-340

Elgar MA, Nash DR (1988) Sexual cannibalism in the garden spider araneus diadematus. Animal Behaviour 36(5):1511 - 1517

Farkas J, Hagen T (2007) Stability and regularity results for a size-structured population model. Journal of Mathematical Analysis and Applications 328(1):119-136

Gallucci F, Ölafsson E (2007) Cannibalistic behaviour of rock-pool copepods: An experimental approach for space, food and kinship. Journal of Experimental Marine Biology and Ecology 342(2):325 - 331

Hirsch MW, Smale S (1974) Differential equations, dynamical systems, and linear algebra. Academic Press, New York :

Kaewmanee C, Tang I (2003) Cannibalism in an age-structured predator-prey system. Ecological Modelling 167(3):213 - 220 
Kohlmeier C, Ebenhöh W (1995) The stabilizing role of cannibalism in a predator-prey system. Bulletin of Mathematical Biology 57:401-411

Liu W, Xiao D, Yi Y (2003) Relaxation oscillations in a class of predator-prey systems. Journal of Differential Equations 188(1):306 - 331

Magnússon KG (1999) Destabilizing effect of cannibalism on a structured predator-prey system. Mathematical Biosciences 155(1):61 - 75

Maury O, Faugeras B, Shin YJ, Poggiale JC, Ari TB, Marsac F (2007) Modeling environmental effects on the size-structured energy flow through marine ecosystems. part 1: The model. Progress In Oceanography 74(4):479 - 499

O'Malley R (1991) Singular perturbation methods for ordinary differential equations. Applied mathematical sciences, Springer

Riley GA (1946) Factors controlling phytoplankton populations on Georges Bank. Journal of Marine Research 6(1):54-73

Rinaldi S, Muratori S (1992) Slow-fast limit cycles in predator-prey models. Ecological Modelling 61(3-4):287-308

de Roos A, Schellekens T, Kooten TV, Wolfshaar KVD, Claessen D, Persson L (2008) Simplifying a physiologically structured population model to a stage-structured biomass model. Theoretical Population Biology 73(1):47 62

Tuljapurkar S, Caswell H (1997) Structured-population models in marine, terrestrial, and freshwater systems, vol 18. Kluwer Academic Pub 